\title{
ARTICLE OPEN High-harmonic generation from spin-polarised defects in solids
}

\author{
M. S. Mrudul ${ }^{1}$, Nicolas Tancogne-Dejean $\mathbb{D}^{2}$, Angel Rubio $\mathbb{D}^{2,3 *}$ and Gopal Dixit ${ }^{1 *}$
}

The generation of high-order harmonics in gases enabled to probe the attosecond electron dynamics in atoms and molecules with unprecedented resolution. Extending these techniques to solids, which were originally developed for atomic and molecular gases, requires a fundamental understanding of the physics that has been partially addressed theoretically. Here, we employ timedependent density-functional theory to investigate how the electron dynamics resulting in high-harmonic emission in monolayer hexagonal boron nitride is affected by the presence of vacancies. We show how these realistic spin-polarised defects modify the harmonic emission and demonstrate that important differences exist between harmonics from a pristine solid and a defected solid. In particular, we found that the different spin channels are affected differently by the presence of the spin-polarised point defect. Moreover, the localisation of the wavefunction, the geometry of the defect, and the electron-electron interaction are all crucial ingredients to describe high-harmonic generation in defected solids.

npj Computational Materials (2020)6:10; https://doi.org/10.1038/s41524-020-0275-z

\section{INTRODUCTION}

Recent advances in mid-infra-red and terahertz laser sources have demonstrated the generation of non-perturbative high-order harmonics from solids, including semiconductors, dielectrics, and nano-structures below their damage threshold. ${ }^{1-14}$ With the pioneering work of Ghimire et al., ${ }^{2}$ high-harmonic generation (HHG) in solids offers fascinating avenues to probe light-driven electron dynamics in solids on attosecond timescale, ${ }^{15-20}$ and to image energy band-dispersion of solids. ${ }^{6,10,21}$ Moreover, due to the high electron density in solids in comparison to gases, HHG from solids may be superior for higher harmonic yield. Finally, HHG in solids represents an attractive route towards compact table-top light-source for coherent and bright attosecond pulses in the extreme ultraviolet and soft x-ray energy regime. 1,9,22,23

Due to the growth processes, defects are inevitable in real solids. ${ }^{24}$ Defects in materials can appear in the form of vacancies, impurities, interstitials (all of these can be neutral as well as charged), dislocations, etc. Defect-induced microscopic modifications in a material significantly affect on its macroscopic properties. $^{25}$ Electronic, optical, vibrational, structural, and diffusion properties of solids with defects have been thoroughly reviewed over the past century. ${ }^{26-32}$ Defect engineering is used to achieve desirable characteristics for materials, e.g., doping has revolutionised the field of electronics. ${ }^{33}$ Defects can also be highly controlled, and it is possible to create isolated defects such as nitrogen-vacancy defects in diamond ${ }^{34,35}$ or single photon emitters in two-dimensional materials. ${ }^{36}$

The influence of defects in solids is not well explored in strongfield physics. In this work, we aim to address the following questions: Is it possible to observe defect-specific fingerprints in strong-field driven electron dynamics? Or does the electron-electron interaction play a different role for defects and bulk materials? Moreover, some defects are also spin polarised in nature. This raises the question if it is possible to control the electron dynamics for different spin channels independently in a non-magnetic host material. As we will show below, HHG is a unique probe that helps us to explore these interesting questions.
We aim to model strong-field driven electron dynamics in a defected-solid with the least approximations. In this work, we employ $a b$ initio time-dependent density functional theory (TDDFT) to simulate the strong-field driven electron dynamics in defected-solid. ${ }^{37}$ This allows us to come up with theoretical predictions relevant for real experiments on defected-solid without empirical input. There are various theoretical predictions for the HHG from doped semiconductors by using simple onedimensional model Hamiltonians. ${ }^{38-40}$ In ref., ${ }^{38}$ by using TDDFT, it is predicted that there is an enhancement of several orders of magnitude in the efficiency of HHG in a donor-doped semiconductor. Using an independent particle model, Huang et al. contrastingly found that the efficiency of the second plateau from the doped semiconductor is enhanced. ${ }^{39}$ Similar calculations within a tight-binding Anderson model indicates that the disorder may lead to well-resolved peaks in HHG. ${ }^{41}$ The conceptual idea for the tomographic imaging of shallow impurities in solids, within a one-dimensional hydrogenic model, has been developed by Corkum and co-workers. ${ }^{42}$ Even though these pioneering works have shown that defects can influence HHG, many points remain elusive. So far, only model systems in one dimension have been considered, and no investigation of realistic defects (through geometry optimisation and relaxation of atomic forces) has been carried out. Beyond the structural aspect, several other essential aspects need to be investigated in order to obtain a better understanding of HHG in defected-solids such as the importance of electron-electron interaction (that goes beyond single-active electron and independent-particle approximations), the role of the electron's spin, the effect of the symmetry breaking due to the defects, etc. Our present work aims to shed some light on some of these crucial questions. For that, we need to go beyond the onedimensional model Hamiltonians.

In order to investigate how the presence of defects modifies HHG in periodic materials, we need to select some systems of interest. Nowadays, two-dimensional (2D) materials are at the centre of tremendous research activities as they reveal different electronic and optical properties compared to the bulk solids. Monolayer 2D materials such as transition-metal

\footnotetext{
${ }^{1}$ Department of Physics, Indian Institute of Technology Bombay, Powai, Mumbai 400076, India. ${ }^{2}$ Max Planck Institute for the Structure and Dynamics of Matter, Luruper Chaussee 149, 22761 Hamburg, Germany. ${ }^{3}$ Nano-Bio Spectroscopy Group and ETSF, Departamento de Fisica de Materiales, Universidad del País Vasco UPV/EHU, 20018 San Sebastián, Spain. *email: angel.rubio@mpsd.mpg.de; gdixit@phy.iitb.ac.in
} 
dichalcogenides, ${ }^{14,43}$ graphene ${ }^{44,45}$ and hexagonal boron nitride $(\mathrm{h}-\mathrm{BN})^{46-48}$ among others have been used to generate strong-field driven high-order harmonics. Many studies have examined HHG in $\mathrm{h}-\mathrm{BN}$ when the polarisation of the laser pulse is either in-plane ${ }^{47,48}$ or out-of-plane ${ }^{46}$ of the material. Using an out-of-plane driving laser pulse, Tancogne-Dejean et al. have shown that atomic-like harmonics can be generated from h-BN. ${ }^{46}$ Also, h-BN is used to explore the competition between atomic-like and bulk-like characteristics of $\mathrm{HHG}^{46,47}$ In $\mathrm{MoS}_{2}$, it has been demonstrated experimentally that the generation of high-order harmonics is more efficient in a monolayer in comparison to its bulk counterpart. ${ }^{43}$ Moreover, HHG from graphene exhibits an unusual dependence on the laser ellipticity. ${ }^{44}$ Light-driven control over the valley pseudospin in $\mathrm{WSe}_{2}$ is demonstrated by Langer et al. ${ }^{14}$ These works have shed light on the fact that 2D materials are promising for studying light-driven electron dynamics and for more technological applications in petahertz electronics ${ }^{49}$ and valleytronics. $^{50}$

Monolayer h-BN is an interesting material for the study of electronic and optical properties. h-BN is a promising candidate for light-emitting devices in the far UV region due to the strong exciton emission. ${ }^{51,52}$ Due to this technological importance, several experimental and theoretical studies have been carried out for h-BN with defects. ${ }^{36,52-68}$ Different kinds of defects in h-BN can be classified as vacancy (mono-vacancies to cluster of vacancies), antisite, and impurities. In particular, defects like monovacancies of boron and nitrogen atoms in h-BN are among the most commonly observed defects. Recently, Zettili and coworkers have shown the possibility of engineering a cluster of vacancies and characterising them using ultra-high-resolution transmission electron microscopy. ${ }^{56,61,63}$ Signatures of defects in h-BN are identified by analysing cathodoluminescence and photoluminescence spectra. ${ }^{51,64}$ It is shown that the emission band around $4 \mathrm{eV}$ originates from the transitions involving deep defect levels. ${ }^{65}$ Ultra-bright single-photon emission from a single layer of h-BN with nitrogen vacancy is achieved experimentally, for example in large-scale nano-photonics and quantum information processing. ${ }^{36,53}$ In refs., ${ }^{54,65-67,69}$ different kinds of defects in h-BN are modelled, and their effects on the electronic and optical properties are thoroughly investigated.

In the present work, using the well-established supercell approach to model the defects in h-BN, we analyse their influence on HHG. Due to the partial ionic nature of its bonds, h-BN is a wide band-gap semiconductor with an experimental band-gap of $6 \mathrm{eV}$. This makes h-BN an interesting candidate for generating highorder harmonics without damaging the material. Moreover, 2D material like h-BN enable us to easily visualise the induced electron density and localised defect states easily. The presence of boron or nitrogen vacancies in h-BN acts as a spin-polarised defect. These factors make $\mathrm{h}-\mathrm{BN}$ an ideal candidate for exploring spin-resolved HHG in non-magnetic defected solids. Below, we will discuss HHG in h-BN with and without spin-polarised monovacancies.

\section{RESULTS}

\section{Computational approach}

$\mathrm{h}-\mathrm{BN}$ has a two-atoms primitive cell. We model the vacancy in a $5 \times 5(7 \times 7)$ supercell with 50 atoms (98 atoms) (See methods section for the details of structural optimisation) following the methods in ref. ${ }^{54}$ The size of the $5 \times 5$ supercell is large enough to separate the nearest defects with a distance greater than $12 \AA$. This minimises the interaction between the nearby defects, ${ }^{54,69}$ and the defect wavefunctions are found to be well-localised within the supercell. ${ }^{66}$ In the present work, we are not considering more than one point defect. Both of our vacancy configurations (single boron as well as single nitrogen vacancy) have a total magnetic moment of $+1 \mu_{\mathrm{B}}$, which is consistent with the earlier reported results. ${ }^{54,66,69}$ For the low defect-concentration considered here ( 2 and $\sim 1 \%$ ), the strength of the total magnetic moment is independent of the defect concentrations. ${ }^{69}$ We have found that the $7 \times 7$ and $5 \times 5$ supercells converged to similar groundstates. $^{54}$

In all the present calculations, we consider a laser pulse of 15femtosecond duration at full-width half-maximum with sinesquared envelope and a peak intensity of $13.25 \mathrm{TW} / \mathrm{cm}^{2}$ in matter (for an experimental in-plane optical index $n$ of $\sim 2.65^{70}$ ). The carrier wavelength of the pulse is $1600 \mathrm{~nm}$, which corresponds to a photon energy of $0.77 \mathrm{eV}$. The polarisation of the laser is linear and its direction is normal to the mirror plane of $h-B N$. The symmetry of the pristine h-BN permits the observation of the harmonics in the parallel and perpendicular directions to the laser polarisation. The direction resolved analysis of the HHG spectrum is shown in the Supplementary Information. For pristine h-BN, the band gap is found to be $4.73 \mathrm{eV}$ within DFT-PBE level. The energy band-gap falls near the sixth harmonic of the incident photon energy and laser parameters are well-below the damage threshold of the pristine.

High-harmonic generation in hexagonal boron nitride with a boron vacancy

We start our analysis by comparing the HHG from pristine h-BN and from h-BN with a boron vacancy. Removal of a boron atom makes the system spin-polarised. ${ }^{54,69}$ The high-harmonic spectrum of h-BN with a boron vacancy $\left(V_{B}\right)$ and its comparison with the spectrum of pristine h-BN is presented in Fig. 1a. The spectrum of $V_{B}$ is different from the pristine h-BN as evident from the figure. There are two distinct differences: first, the below band-gap harmonics corresponding to $V_{B}$ are significantly enhanced (see Fig. 1c). Second, the harmonics, closer to the energy cutoff, have a much lower yield for $V_{B}$ in comparison to the pristine material.

An interesting aspect for the spin-polarised defects in a nonmagnetic host is that the effect of the defect states, compared to bulk states, can be identified clearly by examining the spinresolved spectrum. The harmonics corresponding to spin-up and spin-down channels in $V_{B}$ are shown in Fig. $1 \mathrm{~b}$. As reflected in the figure, the below band-gap harmonics are different for both the channels. The strength of the third harmonic corresponding to the spin-down channel is much stronger (by an order of magnitude) in comparison to the associated spin-up channel as evident from Fig. 1d. This indicates that the increase in the yield observed in Fig. 1a-c predominantly originates from the spindown channel. At variance, the decrease in harmonic yield in higher energies matches well for both the spin-channels. This is a strong indication that the defect states do not play a direct role in this part of the HHG spectrum of $V_{\mathrm{B}}$, but instead that bulk bands predominantly affect this spectral region, as discussed below.

\section{Gap states and electron dynamics}

To understand the difference in the harmonics yield associated with spin-up and spin-down channels, let us analyse the groundstate energy band-structure. The unfolded band-structures of $V_{B}$ for spin-up and spin-down channels are shown in Fig. 2a, d, respectively. The band-structure within the band-gap region is zoomed for both the channels and shown in Fig. 2b, c. As visible from the figure, there is one spin-up (labelled as 1 in Fig. 2b) and two spin-down (labelled as 2 and 3 in Fig. 2c) defect levels within the band-gap of pristine h-BN. One defect state corresponding to the spin-up channel is pushed within the valence bands (see Fig. 2a). All three defect states within the band-gap are found to be unoccupied. These factors make the $V_{B}$ a triple acceptor. The corresponding wavefunctions for these defect states are presented in Fig. 2e-g. The wavefunctions are found to be localised around the vacancy as expected for dispersionless states. The 

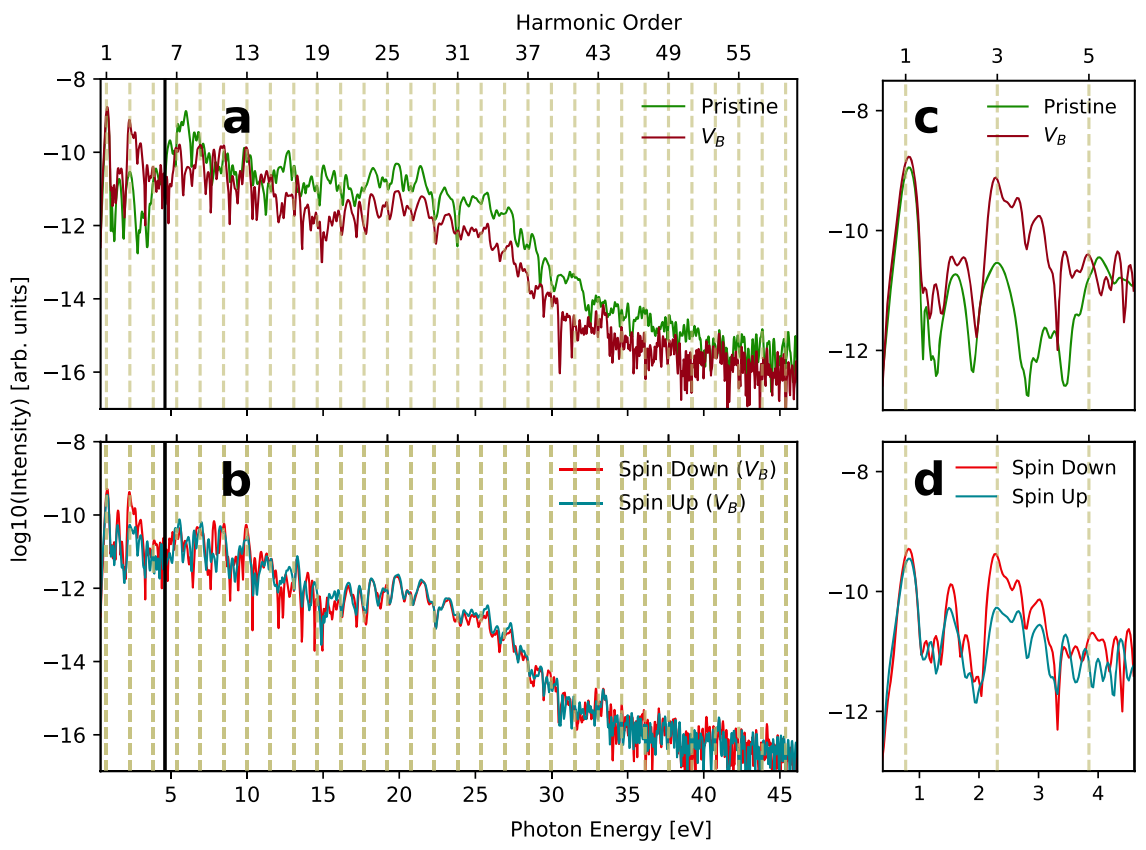

Fig. 1 High-order harmonic spectrum of monolayer hexagonal boron nitride (h-BN) with a boron vacancy. a The total harmonic spectrum of boron-vacant h-BN $\left(V_{B}\right)$ and pristine $h-B N$. The black vertical line represents the energy band-gap of h-BN. b Spin-resolved harmonic spectrum of $V_{B}$. C, d correspond, respectively, to the below band-gap harmonics of (a) and (b).

$p_{\mathrm{x}}$ and $p_{\mathrm{y}}$ states of the nitrogen atoms in the vicinity of a boron vacancy contribute to these defect states, giving a $\sigma$-character to the vacancy wavefunctions. ${ }^{69}$

Unlike the pristine h-BN, spin-up and spin-down electrons in $V_{\mathrm{B}}$ see a different band-structure near the band-gap, since the spinresolved in-gap states are different. Therefore, spin-up and spindown electrons evolve differently in the presence of the laser pulse. It means that interband transitions and ionisation involving the defect states will contribute differently to the spectrum. Hence, the spectral enhancement of the third harmonic can be understood as follows: as visible from the spin-down bandstructure, there is an additional defect state near the valence band, which allows spin-down electrons to be ionised or to recombine through multiple channels and contribute more to the third harmonic (see Fig. 1).

It is evident from the band structure that additional defectstates effectively reduce the minimal band gap needed to reach the conduction bands. Due to the relaxation of atoms next to the vacancy, the pristine bands are also slightly modified. However, this modification is found to be negligible compared to the photon energy of the laser and is not further discussed. In order to understand how the presence of the defect states influences the interband-tunnelling, the number of excited electrons during the laser pulse is evaluated (see Fig. 3a). In the presence of defect states, there are mostly two possible ways in which ionisation can be modified compared to the bulk material. A first possibility is the direct ionisation of the defect states if they are occupied, or filling them if they are originally unoccupied. Another possibility is a double sequential ionisation, in which the defect states play an intermediate role in easing the ionisation to the conduction bands. In $V_{\mathrm{B}}$, there is a finite probability of finding the electrons in conduction bands even after the laser pulse is over (see the red curve in Fig. 3a). In contrast to this, the pulse is not able to promote a significant portion of the valence electrons to the conduction bands permanently in the case of pristine h-BN as the band-gap is significantly large (see the black curve in Fig. 3a). More precisely, for the $5 \times 5$ supercell, we found that 1.6 electrons are ionised, compared to 0.25 in the case of the same cell in the pristine material.
To achieve a better understanding of the possible ionisation mechanisms, we also consider the induced electron density $\left(n_{\text {ind }}\right)$ at two different times near the peak of the vector potential (marked as 1 and 2 in Fig. $3 \mathrm{~b}$ ) and at the end of the laser field (Fig. $3 \mathrm{f}, \mathrm{g})$. As reflected in Fig. 3d, e, the spin-polarised induced densities of $V_{B}$ have a pronounced localised component near the defects and resemble the spatial structure of the initially unoccupied defect wavefunctions (see Fig. 2e-g). The induced densities at the end of the vector potential show that electrons remain in the defect states even after the laser pulse is over (see Fig. $3 f, g)$. Considering that the three defect states are originally unoccupied, we cannot conclude that more electrons are ionised to the conduction bands. It is most likely that in-gap defect states are filled during the laser excitation as 1.6 electrons are excited and $V_{\mathrm{B}}$ is a triple acceptor.

Overall, these results show that the electron dynamics in acceptor-doped solids imply a net transfer of population to the originally unoccupied gap states, but for a wide bandgap host no more electrons are promoted to conduction bands. This explains why only the low-order third harmonic is directly affected by the presence of spin-polarised defect states (as evidenced by the spin dependence of the spectrum). The low density of these defect states means that fewer photons are absorbed. We note that the irreversible population change, assisted by the defect states, ultimately implies that more energy is absorbed by the defected solid, which leads to a lower damage threshold in comparison to the pristine. However, the intensity considered here is low enough to see such an effect.

\section{Effect of electron-electron interaction}

We have established so far that the increase of the low-order harmonic yield is compatible with the presence of the defect states in the band gap of h-BN. This is an independent-particle vision, in which we used the ground-state band-structure of $V_{B}$ to explain the observed effect on the HHG spectrum. We now turn our attention to the higher energy harmonics, for which the harmonic yield is decreased. This seems not to be compatible with a simple vision in terms of the single-particle band structure, 


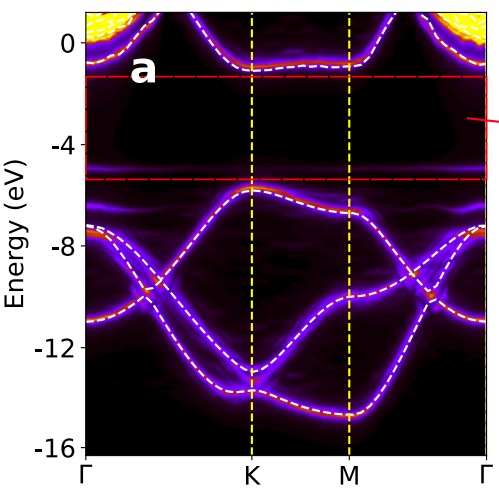

$\mathbf{e}$

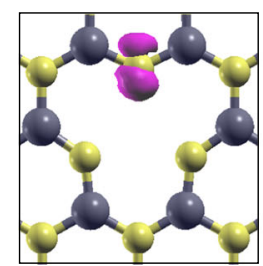

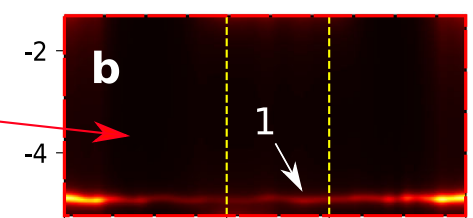

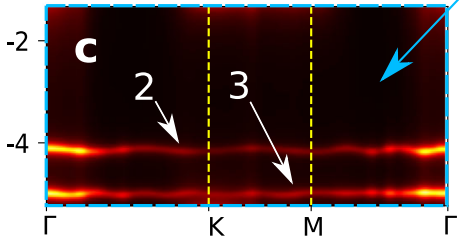

$\mathbf{f}$

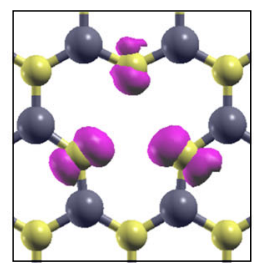

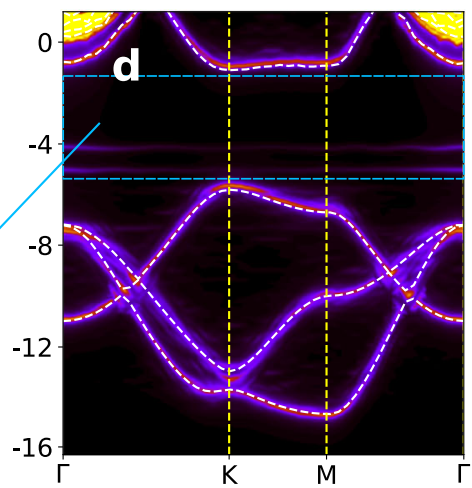

$\mathbf{g}$

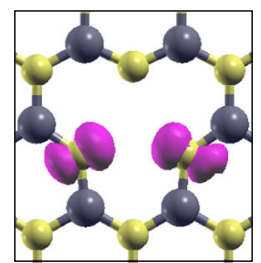

Fig. 2 Spin-resolved unfolded energy band structure and wavefunctions of boron-vacant $\mathbf{h}-\mathbf{B N}\left(\boldsymbol{V}_{\mathbf{B}}\right)$. Spectral function for a spin-up and $\mathbf{d}$ spin-down components of $V_{\mathrm{B}}$. The pristine band-structure is plotted with white dotted lines for reference. The in-gap portion of the spectral function for spin-up and spin-down components are zoomed respectively in $\mathbf{b}$ and $\mathbf{c}$, where vacancy states are recognised by 1 (for spin-up); and by 2 and 3 (for spin-down). e-g The absolute wavefunctions of the in-gap vacancy states as recognised in $\mathbf{b}$ and $\mathbf{c}$, respectively.

especially in view of the fact that more electrons are excited by the laser pulse, as shown in Fig. $3 a$.

To understand this, let us investigate the effect of the electron-electron interaction on the electron dynamics in $V_{B}$ and pristine h-BN. Within the dipole approximation, the HHG spectrum can be expressed as $s^{37,71}$

$\mathrm{HHG}(\omega) \propto\left|\mathcal{F} \mathcal{T}\left[\int d \mathbf{r}\left\{n_{\text {ind }}(\mathbf{r}, t)+n_{0}(\mathbf{r})\right\} \nabla v_{0}(\mathbf{r})\right]+N_{\mathrm{e}} \mathbf{E}(\omega)\right|^{2}$.

Here, $n_{\text {ind }}(\mathbf{r}, t)$ is the induced electron density, $v_{0}$ is the electronnuclei interaction potential, $N_{\mathrm{e}}$ is the total number of electrons, and $\mathbf{E}$ is the applied electric field. $n_{\text {ind }}(\mathbf{r}, t)$ is the difference of the total time-dependent electron density $n(\mathbf{r}, t)$ and the ground-state electron density $n_{0}(\mathbf{r})$, i.e., $n_{\text {ind }}(\mathbf{r}, t)=n(\mathbf{r}, t)-n_{0}(\mathbf{r})$. Also, $n(\mathbf{r}, t)$ is decomposed in spin-polarised fashion as $n(\mathbf{r}, t)=n_{\uparrow}(\mathbf{r}, t)+n_{\downarrow}(\mathbf{r}, t)$. If one analyses this expression, it is straightforward to understand how the introduction of vacancies can change the harmonic spectrum, through a change in the local potential structure near the defect. This results in the change in gradient of the electron-nuclei interaction potential $v_{0}$, which is independent of electrons interacting with each other or not. Apart from this explicit source of change, it is clear that the dynamics of the induced density, evolving from a different ground-state also leads to the modifications in the harmonic spectra. The fact that the ground states are different, and hence $n_{0}(\mathbf{r})$ is different, does not affect the harmonic spectrum because of the absence of time dependence in both $n_{0}$ and $v_{0}$. The possible difference between the HHG spectra can, therefore, be understood in terms of independent particle effects (originating from the structural change of the nuclear potential $v_{0}$ ) and interaction effects from the induced density $n_{\text {ind }}$.

In order to disentangle these two sources of differences between pristine and defect $\mathrm{h}-\mathrm{BN}$, we simulate the harmonic spectra within an independent particle (IP) model by freezing the Hartree and exchange-correlation potentials to the ground-state value. The harmonic spectra for pristine $\mathrm{h}-\mathrm{BN}$ and $V_{\mathrm{B}}$ within TDDFT and IP are compared in Fig. 4. In the case of pristine h-BN, the
HHG-spectra obtained by the IP model and TDDFT are similar. Hence, there is no significant many-body effect in HHG from pristine $\mathrm{h}-\mathrm{BN}$ with an in-plane laser polarisation, at least as described by the PBE functional used here. A similar finding has been reported for $\mathrm{Si}^{37}$ and $\mathrm{MgO}_{1}{ }^{72}$ within the local-density approximation. Only the high-order harmonics display small differences and there the electron-electron interaction reduces the harmonic yield, as found in ref. ${ }^{38}$ In contrast, the HHG-spectra obtained by IP and TDDFT are significantly different for $V_{B}$ as reflected in Fig. 4b. This indicates that the electron-electron interaction is essential for HHG in defected solids. The total currents corresponding to the harmonic spectra for the pristine $h$ $\mathrm{BN}$ and $V_{\mathrm{B}}$ are shown in Supplementary Fig. 2.

For the case of $V_{B}$, the induced electron density is displayed in Fig. 3. This helps us to understand how the spatial structure of the defect wavefunctions influences the spectrum. Similarly, Fig. 3c indicates that the spatial density oscillations are responsible for HHG in pristine h-BN. It is clear that the induced density is different in the two systems. The substantial difference in the harmonic spectra of $V_{B}$ obtained by two approaches, TDDFT and $I P$, is due to the so-called local field effects. This is explored in detail in the following paragraphs. It will be shown that this difference is responsible for the decrease of the harmonic yield for $V_{\mathrm{B}}$.

In the presence of an external electric-field, the localised induced-charge acts as an oscillating dipole near the vacancy. The dipole induces a local electric field, which screens the effect of the external electric field. This is usually referred to as local field effects. The same mechanism is responsible for the appearance of a depolarisation field at the surface of a material driven by an outof-plane electric field. ${ }^{46,47}$ It is important to stress that this induced dipole is expected to play a significant role here, due to the $\sigma$ character of the vacancy wavefunctions. The induced electric field is directly related to the electron-electron interaction term as clearly shown in ref. ${ }^{47}$ As shown in Fig. $4 b$, the harmonic yield at higher energies is increased if we neglect local field effects, i.e., we treat electrons at the IP level. Moreover, the electron-electron interaction also affects the third harmonic, as shown in Fig. 4, but leads to an increase of the harmonic yield. We, therefore, attribute 

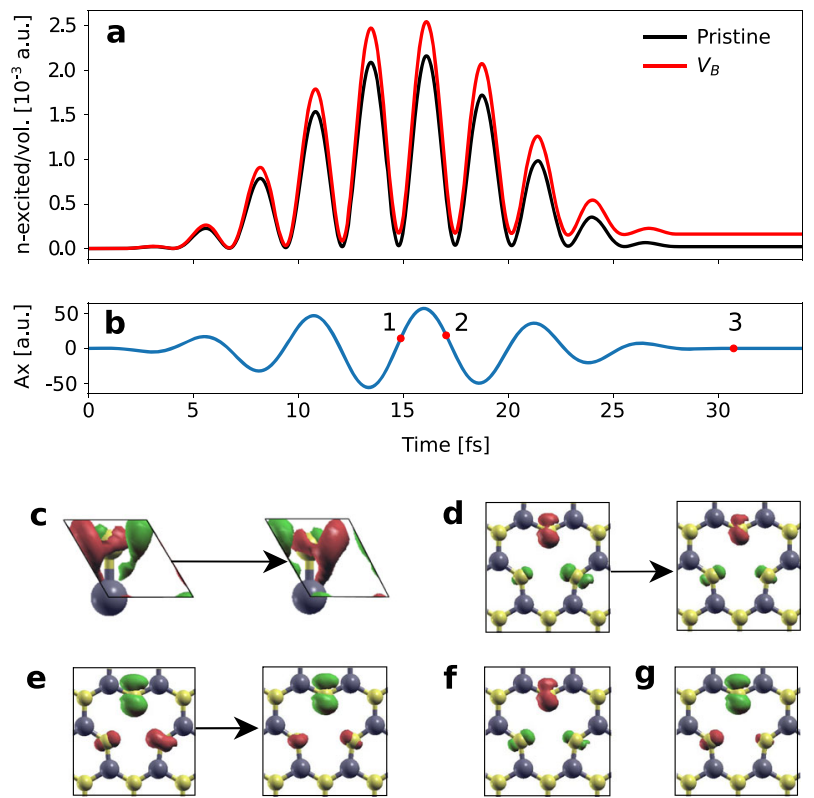

f

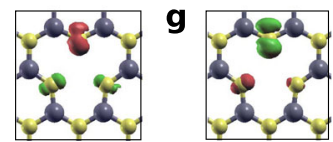

Fig. 3 Time-dependent electron dynamics in boron-vacant h-BN $\left(\boldsymbol{V}_{\mathbf{B}}\right)$. a Number of excited electrons per unit volume in pristine h-BN (black colour) and $V_{B}$ (red colour), and $\mathbf{b}$ vector potential of the driving laser pulse. Snap shots of the time-evolving induced electron density $\left(n_{\text {ind }}\right)$ near the peak of the vector potential [marked as 1 and 2 in b] for c pristine h-BN, d spin-up, and e spin-down channels in $V_{\mathrm{B}}$. $n_{\text {ind }}$ for $\mathbf{f}$ spin-up and $\mathbf{g}$ spin-down channels in $V_{\mathrm{B}}$ after the end of the laser pulse [marked as 3 in b]. Green and red colours in the induced electron density stand for positive and negative values, respectively.

this effect not to local field effects but to correlation effects. It is important to stress that in Maxwell equations, the source term of the induced electric field is the induced density (summed over spin). Therefore, the induced electric field acts equally on the HHG from both spin channels, which is why the decrease of the yield occurs equally on both spin channels, see Fig. $1 \mathrm{~b}$.

We conclude that the modifications in the HHG spectrum due to a point defect originate from a complex interplay of two important factors: the electronic transitions including the in-gap defect states, and the electron-electron interaction. This indicates that HHG in defected-solids cannot be fully addressed via IP approximation as this can lead to wrong qualitative predictions in certain cases.

Finally, we discuss the dependence of the defect concentration on the HHG spectrum by computing the HHG for a $7 \times 7$ supercell with a boron vacancy, which corresponds to a $~ 1 \%$ doping concentration. The harmonic spectrum is presented in Supplementary Fig. 4. The third harmonic enhancement persists with comparable intensity even where there is a lower defect concentration, whereas the higher energy region of the harmonic spectrum matches well with the pristine spectrum. This is consistent with the observation that the higher energy spectrum is dominated by the bulk bands. This indicates that some of the present findings depend on the defect concentration and may not be observed below a certain concentration threshold.

High-harmonic generation in hexagonal boron nitride with a nitrogen vacancy

Until now, we have discussed HHG in h-BN with a boron vacancy. Now we will explore HHG in h-BN with a nitrogen vacancy. Similar to the $V_{\mathrm{B}}$ case, h-BN with a nitrogen vacancy $\left(V_{\mathrm{N}}\right)$ is also spin polarised. Figure 5 a presents the high-order harmonic spectrum of $V_{N}$ and its comparison with the spectrum of pristine h-BN. As apparent from the figure, the spectrum of $V_{N}$ resembles the pristine spectrum more closely, except in the below band-gap regime and for an increase of the yield between 30 and $35 \mathrm{eV}$. All the laser parameters are identical to the case of $V_{B}$. The spectra of $V_{N}$ and $V_{B}$ could be expected to resemble each other as one atom from the pristine h-BN has been removed in both of them. However, this is not the case, as evident from Fig. $5 \mathrm{~b}$ and we note that the high-energy part of the spectrum of $V_{N}$ is much closer to the one of pristine h-BN than $V_{B}$, except above $30 \mathrm{eV}$. The spectra of $V_{N}$ and $V_{B}$ are also fairly different. On close inspection of the below band-gap spectrum, one finds that the third harmonic in $V_{N}$ is significantly enhanced with respect to the pristine case (see Fig. 5c). The same observation was made in the case of HHG in $V_{\mathrm{B}}$. To understand whether the reason behind this enhancement is identical to $V_{B}$ or not, we examined the spin-resolved harmonics in $V_{\mathrm{N}}$. The below band-gap spin-resolved spectrum reveals that the third harmonic has a greater contribution from the spin-up electron than the spin-down electron (see Fig. 5d; also see Supplementary Fig. 5 for the corresponding spectra in the full energy range). This contrasts the findings in $V_{B}$ where the major contribution originated from the spin-down electron. To understand this behaviour, let us explore the ground-state bandstructure of $V_{\mathrm{N}}$.

The unfolded band-structure of $V_{N}$ is presented in Fig. 6. Each of the three boron atoms has an unpaired electron following the removal of a nitrogen atom from pristine h-BN. One spin-up and one spin-down vacancy states emerge within the band-gap and are located more closely to the conduction band (see Fig. 6a-d). One additional defect state is pushed further towards the conduction band in each case. The spin-up defect state is found to be occupied. This makes $V_{\mathrm{N}}$ a single donor. ${ }^{54}$ The $p_{\mathrm{z}}$-states of the boron atoms, which are in the vicinity of a nitrogen vacancy, contribute to the defect states. This gives a $\pi$-character to the defect wavefunctions (Fig. 6e, f). ${ }^{54,66}$ Similar to $V_{B}$, only gap states are analysed. The spin-up defect level is occupied and close to the conduction band, which explains the major contribution of the spin-up electron to the third harmonic in $V_{\mathrm{N}}$. Electrons in this state can easily get ionised to the conduction bands and add more spectral weight to the third harmonic. Note that, unlike $V_{B}$, the local symmetry in $V_{N}$ is preserved, which also explains why the HHG spectrum from $V_{N}$ is close to the HHG from pristine h-BN.

The unfolded band-structures of $V_{N}$ and $V_{B}$ mainly explain the significant enhancement of the third harmonic and its different spin-polarised nature. To explain the overall difference in the harmonic spectrum, the snap-shots of $n_{\text {ind }}$ in $V_{N}$ at different times along the vector potential are presented in Fig. $6 \mathrm{~g}-\mathrm{j}$. In the realspace picture, defect contribution arises from the localised induced density, which can be seen from the integral in Eq. (1). The depletion of the spin-up defect state as well as induced electron density in the spin-down defect state can be observed at the end of the pulse (see Fig. $6 \mathrm{~h}, \mathrm{j}$ ).

In comparison to $V_{B}$, the effect of screening due to the local field effects is weaker in the case of $V_{N}$. In comparison to $V_{B}$, the weaker impact of the local field effects on $V_{N}$ can be attributed to the following two reasons: (1) the induced charge density has a pronounced localised component around the nitrogen vacancy. As evident from Fig. 6e, f, the wavefunctions of spin-up and spindown electrons have similar spatial structure, whereas the corresponding induced charge densities have opposite sign (see Fig. $6 \mathrm{~g}-\mathrm{j}$ ). This partial cancellation of the spin-resolved induced charge density lowers the total induced charge and results in weaker local field effects. (2) For the in-plane laser polarisation, the induced dipole due to the $\pi$-like defect states gets much less polarised than for the $\sigma$-like defect states. This results in weaker screening in $V_{N}$ than $V_{B}$.

The weaker local field effects in $V_{N}$ are fully consistent with the HHG spectra of $V_{N}$, obtained through TDDFT and IP models (see Supplementary Fig. 3). Here, the third harmonic enhancement is 
well captured within the IP model, but the increase in the yield between 30 and $35 \mathrm{eV}$ is found to originate from the electron-electron interaction (see Supplementary Fig. 3). Finally, in the HHG spectrum of $V_{N}$, the defect state plays a role even at higher orders (see the spin-resolved spectra in Supplementary Fig. 5), though these effects are feeble.

In essence, the total HHG spectra corresponding to $V_{B}$ and $V_{N}$ include the contributions from the energy-bands of pristine h-BN

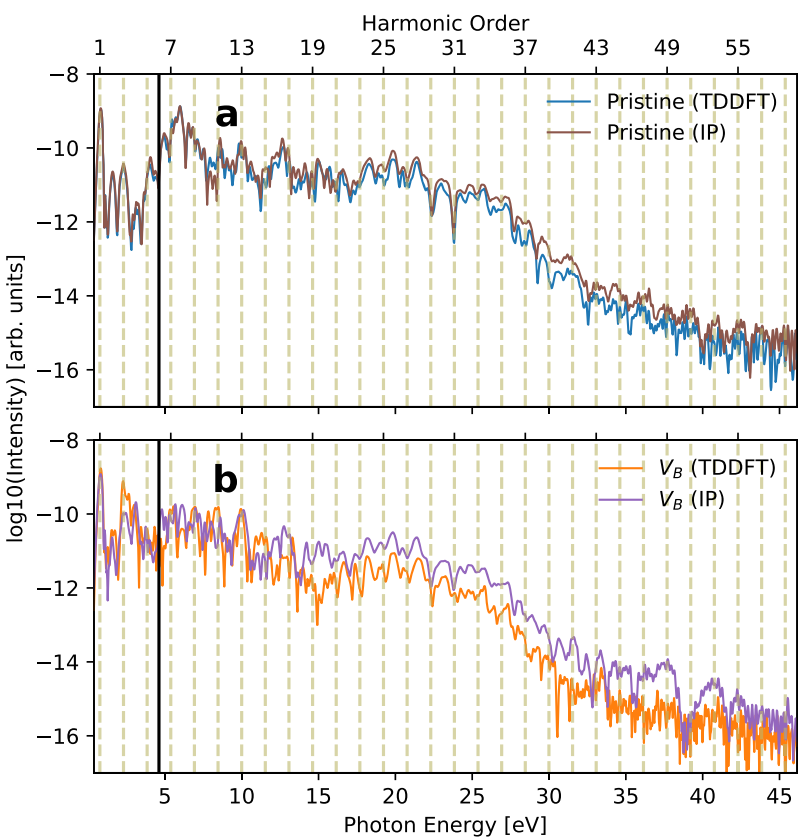

Fig. 4 Many-body effect in harmonic spectrum. a HHG spectrum for pristine h-BN calculated using TDDFT (blue) and the independent particle (IP) approximation (brown). b HHG spectrum for $V_{B}$ calculated using TDDFT (orange) and IP approximation (violet). The black vertical line represents the energy band gap of pristine h-BN. as well as from the transitions including gap states. Moreover, electron-electron interaction plays significant and different roles in both cases. The harmonic spectrum of the defected solid preserves some piece of information of the pristine structure in the higher energy regime along with the characteristic signatures of the defect in the near band-gap regime, or close to the cutoff energy for $V_{\mathrm{N}}$.

\section{DISCUSSION}

In summary, we have investigated the role of vacancy-defect in solid-state HHG. For this purpose, we considered h-BN with a boron or a nitrogen atom vacancy. In simple terms, one may assume that h-BN with a boron atom vacancy or with a nitrogen atom vacancy would exhibit similar HHG spectra since a single atom from h-BN has been removed. However, this is not the case as boron and nitrogen vacancies lead to qualitatively different electronic structures, and this is apparent from their corresponding gap states. It has been found that once an atom is removed from h-BN, either boron or nitrogen, the system becomes spinpolarised with a non-zero magnetic moment near the vacancy. As a consequence, the defect-induced gap states are found to be different for each spin channel and for each vacancy, which we found to be strongly reflected in the low-order harmonics. These contributions are strongly spin-dependent, according to the ordering and occupancy of the defect states. Altogether, the role of the defect states can be understood by analysing the spinpolarised spectra, and the findings are in accordance with the spin polarised band-structure. This establishes one aspect of the role of defect states in strong-field dynamics in solids.

In addition, the vacancy wavefunctions of $V_{B}$ and $V_{N}$ show $\sigma$ and $\pi$-characters, respectively, which lead to different qualitative changes in the harmonic spectra of vacancies, due to the localfield effects and electron correlations. These different behaviours are caused by the creation or absence of an induced dipole, which may counteract the driving electric field, and directly depends on the spatial shape of the defect state wavefunctions. Moreover, the electron-electron interaction also manifests itself in the decrease of the harmonic yield close to the energy cutoff in the $V_{B}$ case,
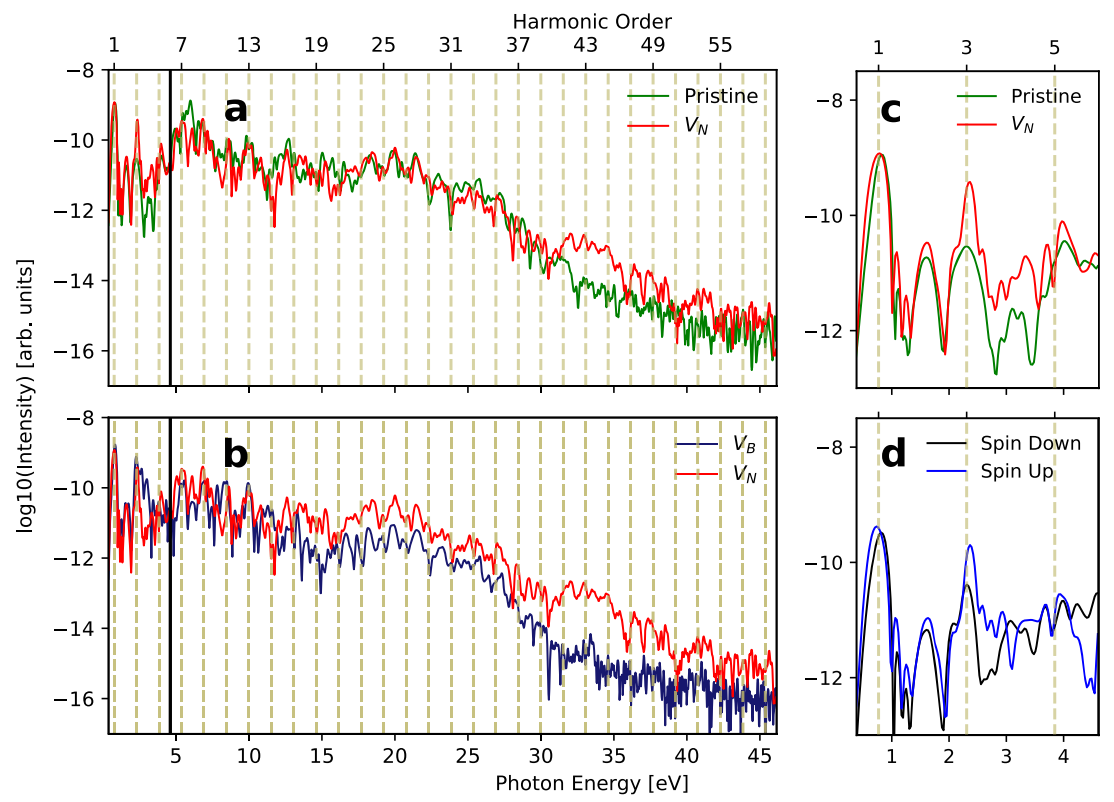

Fig. 5 High-order harmonic spectrum of $\mathbf{h}-\mathrm{BN}$ with nitrogen vacancy. Total harmonic spectrum of a nitrogen vacant h-BN $\left(V_{\mathrm{N}}\right)$ and pristine h-BN, b nitrogen-vacant h-BN $\left(V_{N}\right)$ and boron-vacant h-BN $\left(V_{B}\right)$, and $\mathbf{c}$ below band-gap harmonic spectrum of $V_{N}$ and pristine h-BN. The black vertical line represents the energy band-gap of pristine $\mathrm{h}$-BN. Spin-resolved harmonic spectrum for $\mathbf{d}$ spin-up and e spin-down components, and its comparison with the total below band-gap harmonic spectrum of $V_{\mathrm{N}}$. 

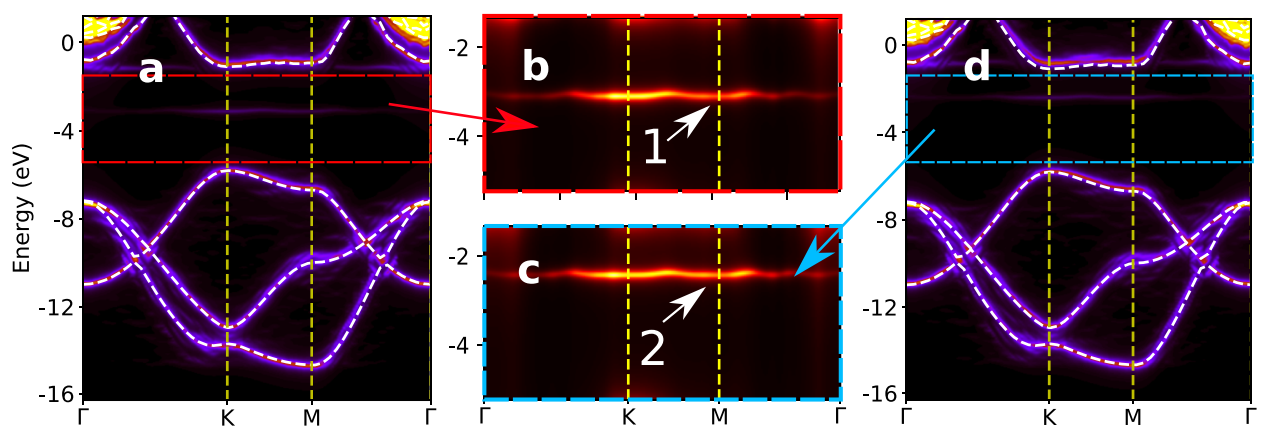

e
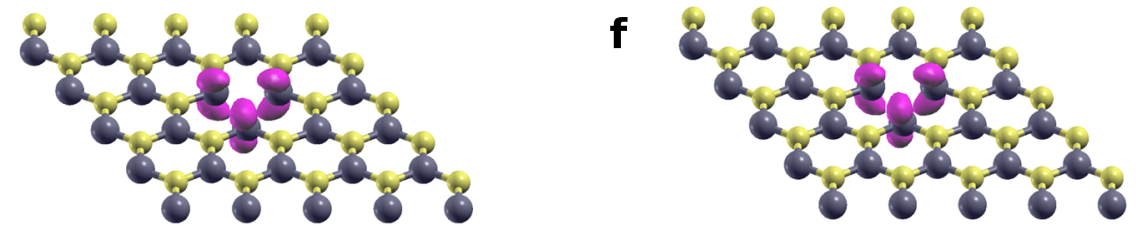

g

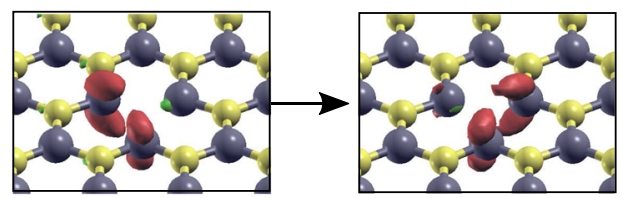

i

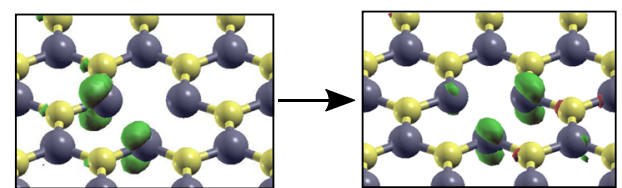

h

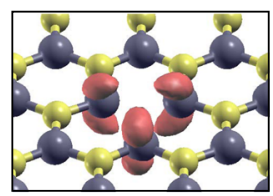

j

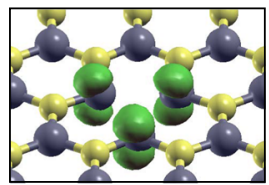

Fig. 6 Spin-resolved unfolded band structure, absolute wavefunctions and induced electron density of nitrogen-vacant $h$-BN ( $V_{\mathrm{N}}$ ). Spectral function for a spin-up and $\mathbf{d}$ spin-down components of $V_{\mathrm{N}}$. The pristine band-structure is plotted with white dashed lines for reference. The in-gap portion of the spectral function for spin-up and spin-down components are zoomed respectively in $\mathbf{b}$ and $\mathbf{c}$ in which the in-gap vacancy states are recognised by 1 (for spin-up); and by 2 (for spin-down). e, $\mathbf{f}$ The absolute wavefunctions of the in-gap vacancy states 1 and $2(\mathbf{a}, \mathbf{b})$, respectively. Snap shots of the time-evolving induced electron density $\left(n_{\text {ind }}\right)$ near the peak of the vector potential for $\mathbf{g}$ spin-up and $\mathbf{i}$ spin-down channels; and after the end of the vector potential for $\mathbf{h}$ spin-up and $\mathbf{j}$ spin-down channels in $V_{\mathrm{N}}$. The vector potential is shown in Fig. 3b. Green and red colours in the induced electron density stand for positive and negative values, respectively.

whereas this effect is completely absent in the $V_{N}$ or pristine cases. This implies that the nature of the vacancies in $V_{B}$ and $V_{N}$ is entirely different, as reflected in their HHG spectra, even at a defect concentration as low as $2 \%$. The HHG spectrum of $V_{N}$ is similar to the pristine $\mathrm{h}-\mathrm{BN}$, whereas the spectrum of $V_{\mathrm{B}}$ differs significantly. These effects essentially imply that some defects are more suited to the modification of the HHG spectra of the bulk materials. This in turn opens the door for tuning HHG by engineering defects in solids.

From our work, we can also estimate about other known defects in h-BN. If one considers the doping impurity instead of vacancy, e.g., carbon impurity, the band-structure of h-BN remains spinpolarised in nature near the band gap..$^{54,65}$ If a boron atom is replaced by a carbon atom, one occupied spin-up and one unoccupied spin-down defect levels appear. Both the defect levels are near the conduction band with the wavefunctions contributed from the $p_{\mathrm{z}}$ orbitals of carbon and nearby nitrogen atoms. So, the carbon doping defect is expected to show qualitatively similar behaviour in the HHG spectra as that observed in the case of $V_{\mathrm{N}}$. On the other hand, one occupied spin-up and one unoccupied spin-down states appear near the conduction bands when nitrogen is replaced with a carbon atom. The wavefunction in this case is contributed mostly by the $p_{z}$ orbitals of the carbon as well as nearby boron atoms. In this case, the enhancement in the below band-gap harmonics is expected due to the defect states near the valence bands similar to $V_{B}$. However, the effect of screening is expected to be lower compared to $V_{B}$ as the nature of wavefunctions here is similar to that of $V_{N}$.

In the case of bi-vacancy in h-BN, one occupied defect state exists near the valence band and two unoccupied defect states near the conduction band (see ref. ${ }^{65}$ ). In this situation, if the separation between defect states and the nearby bands is small compared to the photon energy, no significant changes in the below band-gap harmonics are expected.

Let us finally comment on the appealing possibility of performing imaging of spin-polarised defects in solids using HHG. As spin channels are not equivalent in the studied defects, one might think about using circularly polarised pulses to probe each spin channel independently. However, in the context of dilute magnetic impurities, as studied here, a crystal will host as many defects with positive magnetic moments as the negative ones, and the signals for up or down spin channel will appear as identical after macroscopic averaging.

Our work opens up interesting perspectives for further studies on strong-field electron dynamics in two-dimensional and extended systems, especially involving isolated defects. Further work may address the possibility of monitoring electron-impurity scattering using HHG, more complex defects such as bi-vacancies, and a practical scheme for imaging buried defects in solids. 


\section{METHODS}

Geometry relaxation

Geometry optimisation was performed using the DFT code Quantum ESPRESSO. ${ }^{73,74}$ Both the atomic coordinate and the lattice constant relaxation were allowed. Forces were optimised to be below $10^{-3} \mathrm{eV} / \AA$. We used an energy cutoff of $150 \mathrm{Ry}$, and a k-point grid of $10 \times 10 \times 1 \mathbf{k}$-points. We used a vacuum region of more than $20 \AA$ to isolate the monolayer from its periodic copies. The relaxed lattice constants of 12.60 and $12.57 \AA$ for $V_{B}$, and $12.48 \AA$ for $V_{N}$ were obtained while for pristine $\mathrm{h}-\mathrm{BN}$, it was found to be $12.56 \AA$. The structure of $\mathrm{h}$-BN with a boron vacancy was relaxed by lowering the local threefold symmetry. ${ }^{54}$ The lowering of the symmetry with the boron vacancy is attributed to the Jahn-Teller distortion, which is found to be independent of the defect concentration. ${ }^{54,65,69}$

\section{TDDFT simulations}

By propagating the Kohn-Sham equations within TDDFT, the evolution of the time-dependent current is computed by using the Octopus package. $^{75,76}$ TDDFT within generalised gradient approximation (GGA) with exchange and correlations of Perdew-Burke-Ernzerhof (PBE) ${ }^{77}$ is used for all the simulations presented here. The adiabatic approximation is used for all the time-dependent simulations. We used norm-conserving pseudopotentials. The real-space cell was sampled with a grid spacing of $0.18 \AA$, and a $6 \times 6 \mathbf{k}$-points grid was used to sample the $2 \mathrm{D}$ Brillouin zone. The semi-periodic boundary conditions are employed. A simulation box of $74.08 \AA$ along the nonperiodic dimension, which includes $21.17 \AA$ of absorbing regions on each side of the monolayer, is used. The absorbing boundaries are treated using the complex absorbing potential method and the cap height $\mathrm{h}$ is taken as $\mathrm{h}=-1$ atomic units (a.u.) to avoid the reflection error in the spectral region of interest. ${ }^{78}$

Effective single-particle band-structure or spectral function for the vacancy structures is visualised by unfolding the band-structure of $5 \times$ 5 supercell with 50 atoms. ${ }^{79-81}$ The spin-polarised calculations are used to address the spin-polarised vacancies.

The Fourier-transform of the total spin-polarised time-dependent electronic current $\mathbf{j}_{\sigma}(\mathbf{r}, t)$ is used to simulate the HHG spectrum as

$\operatorname{HHG}(\omega)=\left|\mathcal{F} \mathcal{T}\left[\sum_{\sigma=\uparrow, \downarrow} \frac{\partial}{\partial t}\left(\int d \mathbf{r} \mathbf{j}_{\sigma}(\mathbf{r}, t)\right)\right]\right|^{2}$,

where $\mathcal{F} \mathcal{T}$ and $\sigma$ stand for the Fourier-transform and the spin-index, respectively.

The total number of excited electrons is obtained by projecting the time-evolved wavefunctions $\left(\left|\psi_{n}(t)\right\rangle\right)$ on the basis of the ground-state wavefunctions $\left(\left|\psi_{n^{\prime}}^{\mathrm{GS}}\right\rangle\right)$

$N_{\mathrm{ex}}(t)=N_{\mathrm{e}}-\frac{1}{N_{\mathbf{k}}} \sum_{n, n^{\prime}}^{\mathrm{occ}} \sum_{\mathbf{k}}^{\mathrm{BZ}}\left|\left\langle\psi_{n, \mathbf{k}}(t) \mid \psi_{n^{\prime}, \mathbf{k}}^{\mathrm{GS}}\right\rangle\right|^{2}$,

where $N_{\mathrm{e}}$ is the total number of electrons in the system and $N_{\mathbf{k}}$ is the total number of $\mathbf{k}$-points used to sample the BZ. The sum over the band indices $n$ and $n^{\prime}$ run over all occupied states.

\section{DATA AVAILABILITY}

The data that support the findings of this study are available from the corresponding authors upon request, and will be deposited on the NoMaD repository.

\section{CODE AVAILABILITY}

The OCTOPUS code is available from http://www.octopus-code.org.

Received: 13 July 2019; Accepted: 3 January 2020;

Published online: 31 January 2020

\section{REFERENCES}

1. Ghimire, S. \& Reis, D. A. High-harmonic generation from solids. Nat. Phys. 15, 10-16 (2019).

2. Ghimire, S. et al. Observation of high-order harmonic generation in a bulk crystal. Nat. Phys. 7, 138-141 (2011).

3. Ghimire, S. et al. Redshift in the optical absorption of zno single crystals in the presence of an intense midinfrared laser field. Phys. Rev. Lett. 107, 167407 (2011).
4. Zaks, B., Liu, R. B. \& Sherwin, M. S. Experimental observation of electron-hole recollisions. Nature 483, 580-583 (2012).

5. Schubert, O. et al. Sub-cycle control of terahertz high-harmonic generation by dynamical bloch oscillations. Nat. Photonics 8, 119-123 (2014).

6. Vampa, G. et al. All-optical reconstruction of crystal band structure. Phys. Rev. Lett. 115, 193603 (2015).

7. Vampa, G. et al. Linking high harmonics from gases and solids. Nature 522, 462-464 (2015).

8. Hohenleutner, M. et al. Real-time observation of interfering crystal electrons in high-harmonic generation. Nature 523, 572-575 (2015).

9. Luu, T. T. et al. Extreme ultraviolet high-harmonic spectroscopy of solids. Nature 521, 498-502 (2015).

10. Ndabashimiye, G. et al. Solid-state harmonics beyond the atomic limit. Nature 534, 520-523 (2016).

11. You, Y. S. et al. High-harmonic generation in amorphous solids. Nat. Commun. 8, 724 (2017).

12. Lanin, A. A., Stepanov, E. A., Fedotov, A. B. \& Zheltikov, A. M. Mapping the electron band structure by intraband high-harmonic generation in solids. Optica 4, 516-519 (2017).

13. Sivis, M. et al. Tailored semiconductors for high-harmonic optoelectronics. Science 357, 303-306 (2017).

14. Langer, F. et al. Lightwave valleytronics in a monolayer of tungsten diselenide. Nature 557, 76-80 (2018).

15. Silva, R. E. F., Blinov, I. V., Rubtsov, A. N., Smirnova, O. \& Ivanov, M. High-harmonic spectroscopy of ultrafast many-body dynamics in strongly correlated systems. Nat. Photonics 12, 266-270 (2018).

16. Silva, R. E. F., Jiménez-Galán, Á., Amorim, B., Smirnova, O. \& Ivanov, M. All optical ultrafast probe of a topological phase transition. arXiv preprint arXiv:1806.11232 (2018).

17. Bauer, D. \& Hansen, K. K. High-harmonic generation in solids with and without topological edge states. Phys. Rev. Lett. 120, 177401 (2018).

18. Chacón, A. et al. Observing topological phase transitions with high harmonic generation. arXiv preprint arXiv:1807.01616 (2018).

19. Reimann, J. et al. Subcycle observation of lightwave-driven dirac currents in a topological surface band. Nature 562, 396-400 (2018).

20. Floss, I. et al. Ab initio multiscale simulation of high-order harmonic generation in solids. Phys. Rev. A 97, 011401 (2018).

21. Lanin, A. A., Stepanov, E. A., Fedotov, A. B. \& Zheltikov, A. M. Mapping the electron band structure by intraband high-harmonic generation in solids. Optica 4, 516-519 (2017).

22. Vampa, G. \& Brabec, T. Merge of high harmonic generation from gases and solids and its implications for attosecond science. J. Phys. B 50, 083001 (2017).

23. Kruchinin, S. Y., Krausz, F. \& Yakovlev, V. S. Colloquium: strong-field phenomena in periodic systems. Rev. Mod. Phys. 90, 021002 (2018).

24. Hayes, W. \& Stoneham, A. M. Defects and Defect Processes in Nonmetallic Solids (Courier Corporation, 2012).

25. Wilson, A. H. The theory of electronic semi-conductors.-ii. Proc. R. Soc. Lond. Ser. A Contain. Pap. Math. Phys. Charac. 134, 277-287 (1931).

26. Barker, A. S. Jr \& Sievers, A. J. Optical studies of the vibrational properties of disordered solids. Rev. Mod. Phys. 47, S1 (1975).

27. Pantelides, S. T. The electronic structure of impurities and other point defects in semiconductors. Rev. Mod. Phys. 50, 797 (1978).

28. Queisser, H. J. \& Haller, E. E. Defects in semiconductors: some fatal, some vital. Science 281, 945-950 (1998).

29. Van de Walle, C. G. \& Neugebauer, J. First-principles calculations for defects and impurities: applications to iii-nitrides. J. Appl. Phys. 95, 3851-3879 (2004).

30. Bockstedte, M., Marini, A., Pankratov, O. \& Rubio, A. Many-body effects in the excitation spectrum of a defect in sic. Phys. Rev. Lett. 105, 026401 (2010).

31. Alkauskas, A., Deák, P., Neugebauer, J., Pasquarello, A. \& Van de Walle, C. G. Advanced Calculations for Defects in Materials: Electronic Structure Methods (Wiley, 2011).

32. Freysoldt, C. et al. First-principles calculations for point defects in solids. Rev. Mod. Phys. 86, 253 (2014).

33. Bardeen, J. \& Brattain, W. H. Physical principles involved in transistor action. Phys. Rev. 75, 1208 (1949).

34. Doherty, M. W. et al. The nitrogen-vacancy colour centre in diamond. Phys. Rep. 528, 1-45 (2013).

35. Maze, J. R. et al. Nanoscale magnetic sensing with an individual electronic spin in diamond. Nature 455, 644-647 (2008).

36. Tran, T. T., Bray, K., Ford, M. J., Toth, M. \& Aharonovich, I. Quantum emission from hexagonal boron nitride monolayers. Nat. Nanotechnol. 11, 37-41 (2016).

37. Tancogne-Dejean, N., Mücke, O. D., Kärtner, F. X. \& Rubio, A. Impact of the electronic band structure in high-harmonic generation spectra of solids. Phys. Rev. Lett. 118, 087403 (2017). 
38. Yu, C., Hansen, K. K. \& Madsen, L. B. Enhanced high-order harmonic generation in donor-doped band-gap materials. Phys. Rev. A 99, 013435 (2019).

39. Huang, T. et al. High-order-harmonic generation of a doped semiconductor. Phys. Rev. A 96, 043425 (2017)

40. Pattanayak, A., Mrudul, M. S. \& Dixit, G. Influence of vacancy-defect in solid highharmonic generation. Phys. Rev. A 101, 013404 (2020).

41. Orlando, G., Wang, C. M., Ho, T. S. \& Chu, S. I. High-order harmonic generation in disordered semiconductors. JOSA B 35, 680-688 (2018).

42. Almalki, S. et al. High harmonic generation tomography of impurities in solids: Conceptual analysis. Phys. Rev. B 98, 144307 (2018).

43. Liu, H. et al. High-harmonic generation from an atomically thin semiconductor. Nat. Phys. 13, 262-265 (2017).

44. Yoshikawa, N., Tamaya, T. \& Tanaka, K. High-harmonic generation in graphene enhanced by elliptically polarized light excitation. Science 356, 736-738 (2017).

45. Al-Naib, I., Sipe, J. E. \& Dignam, M. M. High harmonic generation in undoped graphene: interplay of inter-and intraband dynamics. Phys. Rev. B 90, 245423 (2014).

46. Tancogne-Dejean, N. \& Rubio, A. Atomic-like high-harmonic generation from twodimensional materials. Sci. Adv. 4, eaao5207 (2018)

47. Le Breton, G., Rubio, A. \& Tancogne-Dejean, N. High-harmonic generation from few-layer hexagonal boron nitride: evolution from monolayer to bulk response. Phys. Rev. B 98, 165308 (2018).

48. Yu, C. et al. Two-dimensional imaging of energy bands from crystal orientation dependent higher-order harmonic spectra in h-bn. Phys. Rev. B 98, 085439 (2018).

49. Garg, M. et al. Multi-petahertz electronic metrology. Nature 538, 359-363 (2016).

50. Schaibley, J. R. et al. Valleytronics in 2d materials. Nat. Rev. Mater. 1, 16055 (2016).

51. Bourrellier, R. et al. Nanometric resolved luminescence in h-bn flakes: excitons and stacking order. ACS Photonics 1, 857-862 (2014).

52. Bourrellier, R. et al. Bright uv single photon emission at point defects in h-bn. Nano Lett. 16, 4317-4321 (2016).

53. Tran, T. T. et al. Robust multicolor single photon emission from point defects in hexagonal boron nitride. ACS Nano 10, 7331-7338 (2016).

54. Huang, B. \& Lee, H. Defect and impurity properties of hexagonal boron nitride: a first-principles calculation. Phys. Rev. B 86, 245406 (2012)

55. Zobelli, A., Ewels, C. P., Gloter, A. \& Seifert, G. Vacancy migration in hexagonal boron nitride. Phys. Rev. B 75, 094104 (2007).

56. Alem, N. et al. Atomically thin hexagonal boron nitride probed by ultrahighresolution transmission electron microscopy. Phys. Rev. B 80, 155425 (2009).

57. Jiménez, I. et al. Near-edge x-ray absorption fine structure study of bonding modifications in bn thin films by ion implantation. Appl. Phys. Lett. 68, 2816-2818 (1996).

58. Suenaga, K., Kobayashi, H. \& Koshino, M. Core-level spectroscopy of point defects in single layer h-bn. Phys. Rev. Lett. 108, 075501 (2012).

59. Liu, P. et al. Direct observation of defects in hexagonal boron nitride monolayers. Microsc. Microanal. 20, 1738-1739 (2014).

60. Thomas, S., Ajith, K., Chandra, S. \& Valsakumar, M. Temperature dependent structural properties and bending rigidity of pristine and defective hexagonal boron nitride. J. Phys. 27, 315302 (2015).

61. Gilbert, S. M. et al. Fabrication of subnanometer-precision nanopores in hexagonal boron nitride. Sci. Rep. 7, 15096 (2017).

62. Alem, N. et al. Probing the out-of-plane distortion of single point defects in atomically thin hexagonal boron nitride at the picometer scale. Phys. Rev. Lett. 106, 126102 (2011)

63. Wong, D. et al. Characterization and manipulation of individual defects in insulating hexagonal boron nitride using scanning tunnelling microscopy. Nat. Nanotechnol. 10, 949-953 (2015).

64. Pierret, A. et al. Excitonic recombinations in h-bn: from bulk to exfoliated layers Phys. Rev. B 89, 035414 (2014).

65. Attaccalite, C., Bockstedte, M., Marini, A., Rubio, A. \& Wirtz, L. Coupling of excitons and defect states in boron-nitride nanostructures. Phys. Rev. B 83, 144115 (2011).

66. Azevedo, S., Kaschny, J. R., De Castilho, C. M. C. \& de Brito Mota, F. Electronic structure of defects in a boron nitride monolayer. Eur. Phys. J. B 67, 507-512 (2009).

67. Orellana, W. \& Chacham, H. Stability of native defects in hexagonal and cubic boron nitride. Phys. Rev. B 63, 125205 (2001).

68. Mosuang, T. E. \& Lowther, J. E. Influence of defects on the h- bn to c- bn transformation. Phys. Rev. B 66, 014112 (2002).

69. Liu, R.-F. \& Cheng, C. Ab initio studies of possible magnetism in a bn sheet by nonmagnetic impurities and vacancies. Phys. Rev. B 76, 014405 (2007).

70. Geick, R., Perry, C. H. \& Rupprecht, G. Normal modes in hexagonal boron nitride. Phys. Rev. 146, 543-547 (1966).

71. Stefanucci, G. \& Van Leeuwen, R. Nonequilibrium Many-Body Theory of Quantum Systems: A Modern Introduction (Cambridge University Press, Cambridge, 2013).
72. Tancogne-Dejean, N., Mücke, O. D., Kärtner, F. X. \& Rubio, A. Ellipticity dependence of high-harmonic generation in solids originating from coupled intraband and interband dynamics. Nat. Commun. 8, 745 (2017).

73. Giannozzi, P. et al. Quantum espresso: a modular and open-source software project for quantum simulations of materials. J. Phys. 21, 395502 (2009).

74. Giannozzi, P. et al. Advanced capabilities for materials modelling with quantum espresso. J. Phys. 29, 465901 (2017).

75. Andrade, X. et al. Real-space grids and the octopus code as tools for the development of new simulation approaches for electronic systems. Phys. Chem. Chem. Phys. 17, 31371-31396 (2015).

76. Castro, A., Marques, M. A. L. \& Rubio, A. Propagators for the time-dependent kohn-sham equations. J. Chem. Phys. 121, 3425-3433 (2004).

77. Perdew, J. P., Burke, K. \& Ernzerhof, M. Generalized gradient approximation made simple. Phys. Rev. Lett. 77, 3865 (1996).

78. De Giovannini, U., Larsen, A. H. \& Rubio, A. Modeling electron dynamics coupled to continuum states in finite volumes with absorbing boundaries. Eur. Phys. J. B 88, 56 (2015).

79. Ku, W., Berlijn, T. \& Lee, C. C. Unfolding first-principles band structures. Phys. Rev. Lett. 104, 216401 (2010).

80. Popescu, V. \& Zunger, A. Extracting e versus $k$ effective band structure from supercell calculations on alloys and impurities. Phys. Rev. B 85, 085201 (2012).

81. Medeiros, P. V. C., Stafström, S. \& Björk, J. Effects of extrinsic and intrinsic perturbations on the electronic structure of graphene: retaining an effective primitive cell band structure by band unfolding. Phys. Rev. B 89, 041407 (2014).

\section{ACKNOWLEDGEMENTS}

This work was supported by the European Research Council (ERC-2015-AdG694097), the Cluster of Excellence (AIM), Grupos Consolidados (IT1249-19), SFB925, the Flatiron Institute (a division of the Simons Foundation), and Ramanujan fellowship (SB/S2/ RJN-152/2015)

\section{AUTHOR CONTRIBUTIONS}

N.T.-D., A.R., and G.D. conceived the idea, designed the research and supervise the work. M.M.S. performed all the calculations. All authors discussed the results and contributed to the final manuscript.

\section{COMPETING INTERESTS}

The authors declare no competing interests.

\section{ADDITIONAL INFORMATION}

Supplementary information is available for this paper at https://doi.org/10.1038/ s41524-020-0275-z.

Correspondence and requests for materials should be addressed to A.R. or G.D.

Reprints and permission information is available at http://www.nature.com/ reprints

Publisher's note Springer Nature remains neutral with regard to jurisdictional claims in published maps and institutional affiliations.

Open Access This article is licensed under a Creative Commons Attribution 4.0 International License, which permits use, sharing, adaptation, distribution and reproduction in any medium or format, as long as you give appropriate credit to the original author(s) and the source, provide a link to the Creative Commons license, and indicate if changes were made. The images or other third party material in this article are included in the article's Creative Commons license, unless indicated otherwise in a credit line to the material. If material is not included in the article's Creative Commons license and your intended use is not permitted by statutory regulation or exceeds the permitted use, you will need to obtain permission directly from the copyright holder. To view a copy of this license, visit http://creativecommons. org/licenses/by/4.0/.

(c) The Author(s) 2020 